\title{
Counting distinct zeros of the Riemann zeta-function
}

\author{
David W. Farmer
}

Submitted: December 1, 1994; Accepted: December 13, 1994.

ABSTRACT. Bounds on the number of simple zeros of the derivatives of a function are used to give bounds on the number of distinct zeros of the function.

The Riemann $\xi$-function is defined by $\xi(s)=H(s) \zeta(s)$, where $H(s)=\frac{1}{2} s(s-1) \pi^{-\frac{1}{2} s} \Gamma\left(\frac{1}{2} s\right)$ and $\zeta(s)$ is the Riemann $\zeta$-function. The zeros of $\xi(s)$ and its derivatives are all located in the critical strip $0<\sigma<1$, where $s=\sigma+i t$. Since $H(s)$ is regular and nonzero for $\sigma>0$, the nontrivial zeros of $\zeta(s)$ exactly correspond to those of $\xi(s)$. Let $\rho^{(j)}=\beta+i \gamma$ denote a zero of the $j^{\text {th }}$ derivative $\xi^{(j)}(s)$, and denote its multiplicity by $m(\gamma)$. Define the following counting functions:

$$
\begin{array}{rlrl}
N^{(j)}(T) & =\sum_{\rho^{(j)}=\beta+i \gamma} 1 & \text { zeros of } \xi^{(j)}(\sigma+i t) \text { with } 0<t<T \\
N(T) & =N^{(0)}(T) & & \text { zeros of } \xi(\sigma+i t) \text { with } 0<t<T \\
N_{s}^{(j)}(T) & =\sum_{\substack{\rho^{(j)}=\beta+i \gamma \\
m(\gamma)=1}} 1 & \text { simple zeros of } \xi^{(j)}(\sigma+i t) \text { with } 0<t<T \\
N_{s, \frac{1}{2}}^{(j)}(T) & =\sum_{\substack{\rho^{(j)}=\frac{1}{2}+i \gamma \\
m(\gamma)=1}} 1 & \text { simple zeros of } \xi^{(j)}\left(\frac{1}{2}+i t\right) \text { with } 0<t<T \\
M_{r}(T) & =\sum_{\substack{\rho^{(0)}=\beta+i \gamma \\
m(\gamma)=r}} 1 & \text { zeros of } \xi(\sigma+i t) \text { of multiplicity } r \text { with } 0<t<T \\
M_{\leq r}(T) & =\sum_{\substack{\rho^{(0)}=\beta+i \gamma \\
m(\gamma) \leq r}} 1 & \text { zeros of } \xi(\sigma+i t) \text { of multiplicity } \leq r \text { with } 0<t<T
\end{array}
$$

where all sums are over $0<\gamma<T$, and zeros are counted according to their multiplicity. It is well known that $N^{(j)}(T) \sim \frac{1}{2 \pi} T \log T$. Let

$$
\alpha_{j}=\liminf _{T \rightarrow \infty} \frac{N_{s, \frac{1}{2}}^{(j)}(T)}{N^{(j)}(T)} . \quad \quad \beta_{j}=\liminf _{T \rightarrow \infty} \frac{N_{s}^{(j)}(T)}{N^{(j)}(T)}
$$

Thus, $\beta_{j}$ is the proportion of zeros of $\xi^{(j)}(s)$ which are simple, and $\alpha_{j}$ is the proportion which are simple and on the critical line. The best currently available bounds are $\alpha_{0}>0.40219, \alpha_{1}>0.79874$, $\alpha_{2}>0.93469, \alpha_{3}>0.9673, \alpha_{4}>0.98006$, and $\alpha_{5}>0.9863$. These bounds were obtained by combining Theorem 2 of [C2] with the methods of [C1]. Trivially, $\beta_{j} \geq \alpha_{j}$. 
Let $N_{d}(T)$ be the number of distinct zeros of $\xi(s)$ in the region $0<t<T$. That is,

$$
N_{d}(T)=\sum_{n=1}^{\infty} \frac{M_{n}(T)}{n} .
$$

It is conjectured that all of the zeros of $\xi(s)$ are distinct: $N_{d}(T)=N(T)$, or equivalently, all of the zeros are simple: $N_{s}^{(0)}(T)=N(T)$. From the bound on $\alpha_{0}$ we have $N_{s}^{(0)}(T)>\kappa N(T)$, with $\kappa=0.40219$. We will use the bounds on $\beta_{j}$ to obtain the following

Theorem. For $T$ sufficiently large,

$$
N_{d}(T)>k N(T)
$$

with $k=0.63952 \ldots$ Furthermore, given the bounds on $\beta_{j}$, this result is best possible.

We present two methods for determining lower bounds for $N_{d}(T)$. These methods employ combinatorial arguments involving the $\beta_{j}$. We note that the added information that $\alpha_{j}$ detects zeros on the critical line is of no use in improving our result. Everything below is phrased in terms of the Riemann $\xi$-function, but the manipulations work equally well for any function such that it and all of its derivatives have the same number of zeros. We write $f(T) \gtrsim g(T)$ for $f(T) \geq g(T)+o(N(T))$ as $T \rightarrow \infty$. For example, $N_{s}^{(j)}(T) \gtrsim \beta_{j} N(T)$ means $N_{s}^{(j)}(T) \geq\left(\beta_{j}+o(1)\right) N(T)$ as $T \rightarrow \infty$.

The first method starts with the following inequality of Conrey, Ghosh, and Gonek [CGG]. A simple counting argument yields

$$
N_{d}(T) \geq \sum_{r=1}^{R} \frac{M_{\leq r}(T)}{r(r+1)}+\frac{M_{\leq R+1}(T)}{R+1}
$$

To obtain lower bounds for $M_{\leq r}(T)$ we note that if $\rho$ is a zero of $\xi(s)$ of order $m \geq n+2$ then $\rho$ is a zero of order $m-n \geq 2 m /(n+2) \geq 2$ for $\xi^{(n)}(s)$. Thus,

$$
N_{s}^{(n)}(T) \leq N(T)-\frac{2}{n+2}\left(N(T)-M_{\leq n+1}(T)\right),
$$

which gives

$$
M_{\leq n}(T) \gtrsim\left(\frac{\beta_{n-1}(n+1)-n+1}{2}\right) N(T) .
$$

The bounds for $\alpha_{j}$ now give: $M_{\leq 1}(T) \gtrsim 0.40219 N(T), M_{\leq 2}(T) \gtrsim 0.69812 N(T), M_{\leq 3}(T) \gtrsim$ $0.86938 N(T), M_{\leq 4}(T) \gtrsim 0.91825 N(T), M_{\leq 5}(T) \gtrsim 0.94019 N(T)$, and $M_{\leq 6}(T) \gtrsim 0.9520 N(T)$. Inserting these bounds into inequality (2) with $R=5$ gives $N_{d}(T) \gtrsim 0.62583 N(T)$. We note that the lower bounds for $M_{\leq n}(T)$ are best possible in the sense that, for each $n$ separately, equality could hold in (3). However, inequality (3) is not simultaneously sharp for all $n$, and this possibility imparts some weakness to the result. A lower bound for $N_{d}(T)$ was calculated in [CGG] in a spirit similar to the above computation, but it was mistakenly assumed that $M_{\leq n}(T) \gtrsim \beta_{n-1} N(T)$, rendering their bound invalid.

Our second method eliminates the loss inherent in the first method. We start with this 
Lemma. In the notation above,

$$
N_{s}^{(n)}(T) \leq \sum_{j=1}^{n+1} M_{j}(T)+n \sum_{j=n+2}^{\infty} \frac{M_{j}(T)}{j}
$$

Proof. Suppose $\rho$ is a zero of order $j$ for $\xi(s)$. If $j \geq n+2$ then $\rho$ is a zero of order $j-n$ for $\xi^{(n)}(s)$, so $\xi^{(n)}(s)$ has at least $\sum_{j=n+2}^{\infty} \frac{(j-n) M_{j}(T)}{j}$ zeros of order $\geq 2$. Thus,

$$
\begin{aligned}
N_{s}^{(n)}(T) & \leq N^{(n)}(T)-\sum_{j=n+2}^{\infty} \frac{(j-n) M_{j}(T)}{j} \\
& =\sum_{j=0}^{\infty} M_{j}(T)-\sum_{j=n+2}^{\infty} \frac{(j-n) M_{j}(T)}{j} \\
& =\sum_{j=1}^{n+1} M_{j}(T)+n \sum_{j=n+2}^{\infty} \frac{M_{j}(T)}{j},
\end{aligned}
$$

as claimed.

Combining the Lemma with (1) we get

$$
N_{s}^{(n)}(T) \leq n N_{d}(T)+n \sum_{j=1}^{n+1}\left(\frac{1}{n}-\frac{1}{j}\right) M_{j}(T)
$$

Let $I_{n}$ denote the inequality (4). Then, in the obvious notation, a straightforward calculation finds that the inequality

$$
I_{J}+\sum_{n=1}^{J-1} 2^{J-n-1} I_{n}
$$

is equivalent to

$$
\left(2^{J}-1\right) N_{d}(T)+\sum_{n=1}^{J+1} \frac{M_{n}(T)}{n} \geq 2^{J-1} M_{1}(T)+N_{s}^{(J)}(T)+\sum_{n=1}^{J-1} 2^{J-n-1} N_{s}^{(n)}(T) .
$$

This implies

$$
\begin{aligned}
N_{d}(T) & \geq 2^{-J}\left(2^{J-1} N_{s}^{(0)}(T)+N_{s}^{(J)}(T)+\sum_{n=1}^{J-1} 2^{J-n-1} N_{s}^{(n)}(T)\right) \\
& \gtrsim 2^{-J}\left(2^{J-1} \beta_{0}+\beta_{J}+\sum_{n=1}^{J-1} 2^{J-n-1} \beta_{n}\right) N(T) .
\end{aligned}
$$

Choose $J=5$ and use the trivial inequality $\beta_{j} \geq \alpha_{j}$ and the bounds for $\alpha_{j}$ to obtain the Theorem. 
Finally, we show that our result is best possible. In other words, if our lower bounds for the $\beta_{j}$ were actually equalities, then the lower bound given by (6) is sharp. We will accomplish this by showing that the $M_{n}(T)$, the number of zeros of $\xi(s)$ with multiplicity exactly $n$, can be assigned values which achieve the bounds on $\beta_{j}$, and which yield a value of $N_{d}(T)$ which is arbitrarily close to the lower bound given by (6).

Suppose we have lower bounds for $\beta_{j}$, for $0 \leq j \leq J$, and let $K \geq J+2$. Suppose we had the following four equalities:

$$
\begin{gathered}
M_{1}(T)=\beta_{0} N(T), \\
M_{K}(T)=\frac{K}{K-J}\left(1-\beta_{J}\right) N(T), \\
M_{J+1}(T)=\frac{J+1}{2}\left(\beta_{J}-\beta_{J-1}-\frac{1-\beta_{J}}{K-J}\right) N(T),
\end{gathered}
$$

and for $2 \leq n \leq J$,

$$
M_{n}(T)=\frac{n}{2}\left(\frac{3 \beta_{n-1}}{2}-\beta_{n-2}-2^{n-J-1} \beta_{J}-\frac{1-\beta_{J}}{2^{J-n+1}(K-J)}-\sum_{j=n}^{J-1} 2^{n-j-2} \beta_{j}\right) N(T)
$$

and $M_{j}(T)=0$ otherwise. Then $\sum_{j=1}^{\infty} M_{j}(T)=N(T)$ and for $0 \leq n \leq J$ we have

$$
\sum_{j=1}^{n+1} M_{j}(T)+n \sum_{j=n+2}^{\infty} \frac{M_{j}(T)}{j}=\beta_{n} N(T)
$$

and

$$
\sum_{n=1}^{\infty} \frac{M_{n}(T)}{n}=2^{-J}\left(2^{J-1} \beta_{0}+\beta_{J}+\sum_{n=1}^{J-1} 2^{J-n-1} \beta_{n}\right) N(T)+\frac{\left(1-\beta_{J}\right) 2^{-J}}{K-J} N(T) .
$$

Since the left side of $(8)$ is $N_{d}(T)$ and the second term on the right side can be made arbitrarily small by choosing $K$ large, we conclude that (6) is sharp. There are two things left to check. The given values of $M_{n}(T)$ must be positive when $K$ is large. It is easy to check this for $J=5$ and our lower bounds for $\beta_{j}$. And since we supposed that our bounds for $\beta_{j}$ are sharp, we must show that $N_{s}^{(j)}(T)=\beta_{j} N(T)$. To see this, note that, generically, the left side of (7) equals $N_{s}^{(j)}(T)$. In other words, the zeros of the derivatives of a generic function are all simple, except for those which are "tied up" in high-order zeros of the original function.

By computing further values of $\alpha_{j}$, enabling us to take a larger value of $J$ in (6), we could improve the result slightly: this is due to a decrease in the loss in passing from (5) to (6). The bound $M_{\leq 6}(T) \gtrsim 0.952 N(T)$ implies that this improvement could increase the lower bound we obtained by at most $0.00021 N(T)$.

\section{References}

[C1] J. B. Conrey, Zeros of derivatives of Riemann's $\xi$-function on the critical line, II, J. Number Theory 17 (1983), 71-75. 
[C2] J. B. Conrey, More than two fifths of the zeros of the Riemann zeta function are on the critical line, J. reine angew. Math. 399 (1989), 1-26.

[CGG] J. B. Conrey, A. Ghosh, and S. M. Gonek, Mean values of the Riemann zeta-function with application to distribution of zeros, Number Theory, Trace Formulas and Discrete Groups, (1989), 185-199.

[L] N. Levinson, More than one-third of the zeros of Riemann's zeta-function are on $\sigma=\frac{1}{2}$, Adv. in Math., 13 (1974), 383-436.

Mathematical Sciences Research Institute

1000 Centennial Drive

Berkeley, CA 94720

farmer@msri.org 